\title{
Scattering of High Speed Particles in the Kerr Gravitational Field
}

\author{
C. Barrabès*and P.A. Hogan ${ }^{\dagger}$ \\ Laboratoire de Mathématiques et Physique Théorique \\ CNRS/UMR 6083, Université F. Rabelais, 37200 TOURS, France
}

\begin{abstract}
We calculate the angles of deflection of high speed particles projected in an arbitrary direction into the Kerr gravitational field. This is done by first calculating the light-like boost of the Kerr gravitational field in an arbitrary direction and then using this boosted gravitational field as an approximation to the gravitational field experienced by a high speed particle. In the rest frame of the Kerr source the angles of deflection experienced by the high speed test particle can then easily be evaluated.
\end{abstract}

\footnotetext{
*E-mail : barrabes@celfi.phys.univ-tours.fr

${ }^{\dagger}$ E-mail : peter.hogan@ucd.ie
} 


\section{Introduction}

The scattering of a high speed particle in the Kerr gravitational field is examined using a novel viewpoint first introduced by the authors in [1]. In the rest frame of the particle the Kerr field is distorted, as the speed of the particle relative to the source of the Kerr field approaches the speed of light. In the light-like limit the Kerr field becomes the field of an impulsive gravitational wave. Thus from the point of view of a particle travelling close to the speed of light the Kerr gravitational field can be approximated by an impulsive gravitational wave field, and the path of the particle calculated using the time-like geodesic equations of this space-time. When the path is expressed in the rest-frame of the Kerr source the angles of deflection can be deduced. While in [1] only the case of a particle projected along a direction parallel to the equatorial plane was considered, we here obtain the angles of deflection for an arbirary initial direction of the particle. The results are most accurate for high speed particles projected with large impact parameter. Under these circumstances the leading terms in the calculated angles of deflection agree with the known angles of deflection of photons in the Kerr field.

In order to examine the scattering of high speed particles projected in an arbitrary direction into the Kerr field from this point of view we first require the light-like boost of the Kerr field in an arbitrary direction. This is given in section 2. The result is presented in a form which is well adapted to the study of high-energy scattering processes such as the one considered in this paper. Another application is the classical production of $D$-dimensional black holes in high-energy collisions 2. In those scenarios the highly relativistic colliding particles are modelled by plane-fronted impulsive gravitational waves, in order to study the formation of a trapped surface after the collision of the

two waves, and derive estimates of the mass of the resulting black hole and of the classical cross-section for black hole production.

\section{Boosted Kerr Field in an Arbitrary Direc- tion}

Our approach to analyzing the scattering of a high speed particle in the Kerr gravitational field, or indeed in the gravitational field of any isolated source, involves first calculating the gravitational field of the isolated source as it appears to an observer moving rectilinearly relative to the source with speed approaching that of light (see [1] and also [3]). To do this for the Kerr field 
we start with the Kerr line-element in the Kerr-Schild form [4], 5]

$$
d s^{2}=d s_{0}^{2}+\frac{2 m \bar{r}^{3}}{\bar{r}^{4}+A^{2} \bar{z}^{2}}\left(\bar{k}_{\mu} d \bar{x}^{\mu}\right)^{2}=\bar{g}_{\mu \nu} d \bar{x}^{\mu} d \bar{x}^{\nu}
$$

with

$$
\begin{aligned}
d s_{0}^{2} & =d \bar{x}^{2}+d \bar{y}^{2}+d \bar{z}^{2}-d \bar{t}^{2}=\bar{\eta}_{\mu \nu} d \bar{x}^{\mu} d \bar{x}^{\nu} \\
\bar{k}_{\mu} d \bar{x}^{\mu} & =\frac{\bar{z}}{\bar{r}} d \bar{z}+\left(\frac{\bar{r} \bar{x}+A \bar{y}}{\bar{r}^{2}+A^{2}}\right) d \bar{x}+\left(\frac{\bar{r} \bar{y}-A \bar{x}}{\bar{r}^{2}+A^{2}}\right) d \bar{y}-d \bar{t}
\end{aligned}
$$

In (2.1) $m$ is the mass and $\mathbf{J}=(0,0, m A)$ is the angular momentum of the source. Also $\bar{r}$ is given in terms of $\bar{x}, \bar{y}, \bar{z}$ by

$$
\frac{\bar{x}^{2}+\bar{y}^{2}}{\bar{r}^{2}+A^{2}}+\frac{\bar{z}^{2}}{\bar{r}^{2}}=1 \text {. }
$$

The bars on coordinates are for convenience and will be removed below. Our approach to calculating the light-like boost of the gravitational fields of isolated sources is centered on the Riemann tensor and thus we require a convenient form of the Riemann tensor for the Kerr field. This is given, in the barred coordinates, by the complex quantity

$$
{ }^{+} \bar{R}_{\mu \nu \rho \sigma}=\bar{R}_{\mu \nu \rho \sigma}+i^{*} \bar{R}_{\mu \nu \rho \sigma}
$$

where Greek indices take values 1, 2, 3, 4 and the star indicates the dual of the Riemann tensor. Thus ${ }^{*} \bar{R}_{\mu \nu \rho \sigma}=\frac{1}{2} \bar{\eta}_{\mu \nu \alpha \beta} \bar{R}_{\rho \sigma}^{\alpha \beta}$ with $\bar{\eta}_{\mu \nu \alpha \beta}=\sqrt{-\bar{g}} \bar{\epsilon}_{\mu \nu \alpha \beta}$ where $\bar{g}$ is the determinant of the metric tensor in the barred coordinates and $\bar{\epsilon}_{\mu \nu \alpha \beta}$ is the Levi-Civita permutation tensor in the barred coordinates. For the Kerr space-time with line-element (2.1) above ${ }^{+} \bar{R}_{\mu \nu \rho \sigma}$ is given by [6]

$$
{ }^{+} \bar{R}_{\mu \nu \rho \sigma}=-\frac{m \bar{r}^{3}}{\left(\bar{r}^{2}+i A \bar{z}\right)^{3}}\left\{\bar{g}_{\mu \nu \rho \sigma}+i \bar{\epsilon}_{\mu \nu \rho \sigma}+3 \bar{W}_{\mu \nu} \bar{W}_{\rho \sigma}\right\},
$$

where

$$
\bar{g}_{\mu \nu \rho \sigma}=\bar{g}_{\mu \rho} \bar{g}_{\nu \sigma}-\bar{g}_{\mu \sigma} \bar{g}_{\nu \rho},
$$

and $\bar{W}_{\mu \nu}$ is given by the 2 -form

$$
\begin{aligned}
\frac{1}{2} \bar{W}_{\mu \nu} d \bar{x}^{\mu} \wedge d \bar{x}^{\nu}= & \frac{\bar{r}}{\bar{r}^{2}+i A \bar{z}}[\bar{x}(d \bar{x} \wedge d \bar{t}-i d \bar{y} \wedge d \bar{z})+\bar{y}(d \bar{y} \wedge d \bar{t} \\
& -i d \bar{z} \wedge d \bar{x})+(\bar{z}+i A)(d \bar{z} \wedge d \bar{t}-i d \bar{x} \wedge d \bar{y})] .(2 .
\end{aligned}
$$


We require the Kerr field when the angular momentum points in an arbitrary direction in space and not in the positive $\bar{z}$-direction as it does here. With $a, b, c$ real numbers such that $\sqrt{a^{2}+b^{2}+c^{2}}=A$ we make the rotation

$$
\begin{aligned}
& \bar{x} \rightarrow-\frac{\left(a c^{2}+b^{2} A\right)}{A\left(b^{2}+c^{2}\right)} \bar{x}-\frac{b c(a-A)}{A\left(b^{2}+c^{2}\right)} \bar{y}+\frac{c}{A} \bar{z}, \\
& \bar{y} \rightarrow-\frac{b c(a-A)}{A\left(b^{2}+c^{2}\right)} \bar{x}-\frac{\left(a b^{2}+c^{2} A\right)}{A\left(b^{2}+c^{2}\right)} \bar{y}+\frac{b}{A} \bar{z}, \\
& \bar{z} \rightarrow \frac{c}{A} \bar{x}+\frac{b}{A} \bar{y}+\frac{a}{A} \bar{z} .
\end{aligned}
$$

Under this rotation $\mathbf{J}=(0,0, m A) \rightarrow(m c, m b, m a)$ so that the angular momentum of the source points in an arbitrary direction relative to the new $\bar{x}, \bar{y}, \bar{z}$ axes. Substituting (2.9) into (2.4) we find that $\bar{r}$ is given in terms of the new $\bar{x}, \bar{y}, \bar{z}$ by

$$
\bar{x}^{2}+\bar{y}^{2}+\bar{z}^{2}+\frac{(c \bar{x}+b \bar{y}+a \bar{z})^{2}}{\bar{r}^{2}}=\bar{r}^{2}+a^{2}+b^{2}+c^{2} .
$$

In addition the following hold under (2.9):

$$
\begin{gathered}
d \bar{x}^{2}+d \bar{y}^{2}+d \bar{z}^{2} \rightarrow d \bar{x}^{2}+d \bar{y}^{2}+d \bar{z}^{2} \\
\bar{x} d \bar{x}+\bar{y} d \bar{y}+(\bar{z}+i A) d \bar{z} \rightarrow(\bar{x}+i c) d \bar{x}+(\bar{y}+i b) d \bar{y}+(\bar{z}+i a) d \bar{z} \\
\bar{y} d \bar{x}-\bar{x} d \bar{y} \rightarrow \frac{(a \bar{y}-b \bar{z})}{A} d \bar{x}+\frac{(c \bar{z}-a \bar{x})}{A} d \bar{y}+\frac{(b \bar{x}-c \bar{y})}{A} d \bar{z}
\end{gathered}
$$

and

$$
\begin{aligned}
\bar{x} d \bar{y} \wedge d \bar{z}+\bar{y} d \bar{z} \wedge d \bar{x} & +(\bar{z}+i A) d \bar{x} \wedge d \bar{y} \rightarrow(\bar{x}+i c) d \bar{y} \wedge d \bar{z} \\
& +(\bar{y}+i b) d \bar{z} \wedge d \bar{x}+(\bar{z}+i a) d \bar{x} \wedge d \bar{y}
\end{aligned}
$$

We can use these results to see the following: Under (2.9) the Kerr metric tensor retains the Kerr-Schild form

$$
\bar{g}_{\mu \nu}=\bar{\eta}_{\mu \nu}+2 H \bar{k}_{\mu} \bar{k}_{\nu},
$$

with $\bar{\eta}_{\mu \nu}=\operatorname{diag}(1,1,1,-1)$,

$$
H=\frac{m \bar{r}^{3}}{\bar{r}^{4}+(\mathbf{a} \cdot \overline{\mathbf{x}})^{2}},
$$

and

$$
\bar{k}_{\mu} d \bar{x}^{\mu}=\frac{(\mathbf{a} \cdot \overline{\mathbf{x}})(\mathbf{a} \cdot d \overline{\mathbf{x}})}{\bar{r}\left(\bar{r}^{2}+|\mathbf{a}|^{2}\right)}+\frac{\bar{r}(\overline{\mathbf{x}} \cdot \mathbf{d} \mathbf{x})}{\bar{r}^{2}+|\mathbf{a}|^{2}}-\frac{\mathbf{a} \cdot(\overline{\mathbf{x}} \times d \overline{\mathbf{x}})}{\left(\bar{r}^{2}+|\mathbf{a}|^{2}\right)}-d \bar{t}
$$


Here $\mathbf{a}=(c, b, a)$, with $|\mathbf{a}|^{2}=a^{2}+b^{2}+c^{2}$ and $\overline{\mathbf{x}}=(\bar{x}, \bar{y}, \bar{z})$ with $\bar{r}$ given in terms of $\bar{x}, \bar{y}, \bar{z}$ by (2.10). The dot and cross signify the usual scalar and vector product in three dimensional Euclidean space. This form of the Kerr metric has been given by Weinberg [9]. Weinberg's angular momentum points in the opposite direction to ours. Now the Riemann tensor is given by

$$
{ }^{+} \bar{R}_{\mu \nu \rho \sigma}=-\frac{m \bar{r}^{3}}{\left(\bar{r}^{2}+i(\mathbf{a} \cdot \overline{\mathbf{x}})\right)^{3}}\left\{\bar{g}_{\mu \nu \rho \sigma}+i \bar{\epsilon}_{\mu \nu \rho \sigma}+3 \bar{W}_{\mu \nu} \bar{W}_{\rho \sigma}\right\} .
$$

Here $\bar{g}_{\mu \nu \rho \sigma}$ has the form (2.7) with $\bar{g}_{\mu \nu}$ given now by (2.15), the permutation symbol is still $\bar{\epsilon}_{\mu \nu \rho \sigma}$ on account of its transformation properties under a general coordinate transformation (see [10]) and the fact that the Jacobian of the transformation (2.9) is unity. Also $\bar{W}_{\mu \nu}$ in (2.18) is now given by

$$
\begin{aligned}
\frac{1}{2} \bar{W}_{\mu \nu} d \bar{x}^{\mu} \wedge d \bar{x}^{\nu}= & \frac{\bar{x}}{\bar{r}^{2}+i(\mathbf{a} \cdot \overline{\mathbf{x}})}[(\bar{x}+i c)(d \bar{x} \wedge d \bar{t}-i d \bar{y} \wedge d \bar{z}) \\
& +(\bar{y}+i b)(d \bar{y} \wedge d \bar{t}-i d \bar{z} \wedge d \bar{x}) \\
& +(\bar{z}+i a)(d \bar{z} \wedge d \bar{t}-i d \bar{x} \wedge d \bar{y})]
\end{aligned}
$$

We make a Lorentz boost in the $\bar{x}$-direction:

$$
\bar{x}=\gamma(x+v t), \quad \bar{y}=y, \quad \bar{z}=z, \quad \bar{t}=\gamma(t+v x),
$$

with $\gamma=\left(1-v^{2}\right)^{-1 / 2}$. We are using units in which the speed of light is unity. As always in such a boost (see [3, 11]) the mass of the source scales as $m=p \gamma^{-1}$ where $p$ is a constant (the "energy" of the source). Also the components of the angular momentum per unit mass orthogonal to the boost direction (the constants $b$ and $a$ ) remain finite but the component in the direction of the boost scales as $c=\hat{c} \gamma^{-1}$ where $\hat{c}$ is a constant (see [6], section III for an explanation of this). The physical origin of this scaling of $c$ is that the multipole moments of the isolated source of a gravitational field experience a Lorentz contraction in the direction of the boost (see [7]). In the case of the Kerr source the multipole moments are constructed from the mass and the angular momentum per unit mass (see, for example, [6] and [8] ) in such a way that the Lorentz contraction in the direction of the boost is equivalent to the scaling of the component of the angular momentum per unit mass $c$ in that direction given here. We apply (2.20) to the Riemann tensor (2.18) to obtain ${ }^{+} R_{\mu \nu \rho \sigma}$ and then take the limit $v \rightarrow 1$. The result will be the gravitational field of the boosted source in the form

$$
{ }^{+} \tilde{R}_{\mu \nu \rho \sigma}=\lim _{v \rightarrow 1}+R_{\mu \nu \rho \sigma}
$$


To evaluate this limit we shall need generalizations of equations (2.18) and (2.19) in [6]. These generalizations are based on the identity

$$
\frac{\bar{r}^{3}}{\left(\bar{r}^{2}+i(\mathbf{a} \cdot \overline{\mathbf{x}})\right)^{3}}=\frac{1}{\left[(\bar{y}+i b)^{2}+(\bar{z}+i a)^{2}\right]} \frac{\partial}{\partial \bar{x}}\left(\frac{(\bar{x}+i c) \bar{r}}{\bar{r}^{2}+i(\mathbf{a} \cdot \overline{\mathbf{x}})}\right) .
$$

Proceeding as in [6] we derive from this the limit

$$
\lim _{v \rightarrow 1} \frac{\gamma \bar{r}^{3}}{\left(\bar{r}^{2}+i(\mathbf{a} \cdot \overline{\mathbf{x}})\right)^{3}}=\frac{2 \delta(x+t)}{(y+i b)^{2}+(z+i a)^{2}},
$$

where $\delta(x+t)$ is the Dirac delta function singular on $x=-t$. Differentiating (2.23) with respect to $y=\bar{y}$ and using

$$
\frac{\partial \bar{r}}{\partial \bar{y}}=\frac{\bar{r}^{3} \bar{y}+\bar{r} b(\mathbf{a} \cdot \overline{\mathbf{x}})}{\bar{r}^{4}+i(\mathbf{a} \cdot \overline{\mathbf{x}})^{2}}
$$

we arrive at another useful limit:

$$
\lim _{v \rightarrow 1} \frac{\gamma \bar{r}^{5}}{\left(\bar{r}^{2}+i(\mathbf{a} \cdot \overline{\mathbf{x}})\right)^{5}}=\frac{4}{3} \frac{\delta(x+t)}{\left[(y+i b)^{2}+(z+i a)^{2}\right]^{2}} .
$$

As an illustration of (2.21) we find that

$$
\begin{aligned}
{ }^{+} \tilde{R}_{1212} & =4 p\left[\frac{z+i a+i(y+i b)}{(z+i a)^{2}+(y+i b)^{2}}\right]^{2} \delta(x+t) \\
& =4 p\left[\frac{z+b+i(y-a)}{(z+b)^{2}+(y-a)^{2}}\right]^{2} \delta(x+t) .
\end{aligned}
$$

This can be rewritten in the form

$$
{ }^{+} \tilde{R}_{1212}=\left(h_{y y}-i h_{y z}\right) \delta(x+t)
$$

with

$$
h=2 p \log \left\{(y-a)^{2}+(z+b)^{2}\right\} .
$$

Continuing this process for all components of the Riemann tensor we find that $\tilde{R}_{\mu \nu \rho \sigma} \equiv 0$ except for

$$
\begin{aligned}
{ }^{+} \tilde{R}_{1212} & ={ }^{+} \tilde{R}_{2424}=-{ }^{+} \tilde{R}_{1313}=-{ }^{+} \tilde{R}_{3434}=-{ }^{+} \tilde{R}_{3134}={ }^{+} \tilde{R}_{2124} \\
& =\left(h_{y y}-i h_{y z}\right) \delta(x+t)
\end{aligned}
$$

and

$$
\begin{aligned}
{ }^{+} \tilde{R}_{1213} & ={ }^{+} \tilde{R}_{2434}={ }^{+} \tilde{R}_{3124}={ }^{+} \tilde{R}_{2134} \\
& =i\left(h_{y y}-i h_{y z}\right) \delta(x+t)
\end{aligned}
$$


with $h$ given by (2.28). This curvature tensor can be obtained from the metric tensor given via the line-element

$$
d s^{2}=d x^{2}+d y^{2}+d z^{2}-d t^{2}-2 h \delta(x+t)(d x+d t)^{2} .
$$

The process leading to this is described in 3 pp.96 and 97. This curvature tensor describes an impulsive gravitational wave with the null hyperplane $x=-t$ as the history of the wave front. We see from (2.28) that this gravitational field is also singular on $x=-t, y=a, z=-b$. This is a null geodesic generator of the null hyperplane $x=-t$. In the particular case of the boosted Schwarzshild field one recovers the well-known result 11] by simply putting the two angular momentum parameters $b$ and $a$ to zero in (2.28). As noted following eq. (2.21) the angular momentum per unit mass $c$, in the direction of the boost, scales differently from the transverse components $b, a$ in terms of the three-velocity $v$ of the observer, and this explains why $c$ does not appear in the light-like limit $v \rightarrow 1$. This shows that the effect of the introduction of the angular momentum is simply to shift the singularity from $y=z=0$ in the Schwarzschild case, to $y=a, z=-b$

for the Kerr field with angular momentum $\mathbf{J}=(m c, m b, m a)$, the light-like boost being in the $x$-direction.

The light-like boost of the Kerr gravitational field in an arbitrary direction has also been studied from a different point of view in [12. In this work the angle $\alpha$ between the axis of symmetry of the Kerr field and the direction of boost is introduced and the authors conclude that from their point of view "only the limiting cases $\alpha=0$ and $\alpha=\pi / 2 \ldots$ admit a solution in closed form." In addition they point out that in their analysis "the general case allows a perturbative treatment if one suitably rescales the coordinates and expands the resulting expression with respect to $\sin \alpha "$. We have clearly avoided these restrictions by first tilting the axis of the Kerr source and then boosting in the $\bar{x}$-direction. In addition the positive advantage of our Riemann tensor centered approach has already been elucidated in [3] and [6].

\section{Scattering Angles}

We consider the deflection of a highly relativistic particle in the Kerr gravitational field. The direction of the incoming particle is arbitrary. We call $\bar{S}$ the rest frame of the Kerr source and $S$ the rest-frame of the high speed particle. In $S$ the particle sees the Kerr source moving towards it with a speed close to the speed of light. In the ultrarelativistic limit the Kerr gravitational field looks to the particle as the gravitational field of an impulsive gravitational wave. This field is modelled by the space-time with line-element (2.31). In 
$S$ the world-line of the particle is a time-like geodesic of this metric. In $S$ the particle is assumed to be located at $x=0, y=y_{0}, z=z_{0}$ (say). It starts moving after encountering the impulsive gravitational wave. Solving the time-like geodesic equations for the metric given via (2.31) we then have in $S$ the components of the 4-velocity of the particle before and after encountering the impulsive gravitational wave (see [1]). From these the components of the 4-velocity of the particle in $\bar{S}$ are obtained by Lorentz transformation valid for $v$ close to unity. If $\alpha$ is the angle of deflection of the high speed particle out of the $\bar{x} \bar{z}$-plane after encountering the gravitational wave and if $\beta$ is the angle of deflection out of the $\bar{x} \bar{y}$-plane then these angles are given by [1]

$$
\tan \alpha=\frac{\dot{\bar{y}}}{\sqrt{\dot{\bar{x}}^{2}+\dot{\bar{z}}^{2}}}=\frac{Y_{1}}{\left\{Z_{1}^{2}+\gamma^{2}\left[X_{1}(1-v)+v\right]^{2}\right\}^{1 / 2}}
$$

and

$$
\tan \beta=\frac{\dot{\bar{z}}}{\dot{\bar{x}}}=\frac{Z_{1}}{\gamma\left[X_{1}(1-v)+v\right]} .
$$

Here the dot indicates differentiation with respect to proper time and $X_{1}, Y_{1}, Z_{1}$ are calculated from the function $h(y, z)$ according to

$$
X_{1}=-\frac{1}{2}\left[\left(h_{y}\right)_{0}^{2}+\left(h_{z}\right)_{0}^{2}\right], \quad Y_{1}=-\left(h_{y}\right)_{0}, \quad Z_{1}=-\left(h_{z}\right)_{0} .
$$

The brackets around a quantity here followed by a subscript zero denote that the quantity is evaluated at $y=y_{0}, z=z_{0}$. Also (3.1) and (3.2) hold for $v$ close to unity. For the particular function $h$ given by (2.28) the angles of deflection $\alpha$ and $\beta$ are given by

$$
\tan \alpha=\frac{-4 m\left(y_{0}-a\right)}{\left[\left\{\left(y_{0}-a\right)^{2}+\left(z_{0}+b\right)^{2}-4 m^{2}\right\}^{2}+16 m^{2}\left(z_{0}+b\right)^{2}\right]^{1 / 2}},
$$

and

$$
\tan \beta=\frac{-4 m\left(z_{0}+b\right)}{\left(y_{0}-a\right)^{2}+\left(z_{0}+b\right)^{2}-4 m^{2}} .
$$

If the incoming particle is projected from $y_{0}=-\eta(\eta>0), z_{0}=0$ then for large impact parameter $\eta$ these formulas give the small angles

$$
\alpha=\frac{4 m}{\eta}-\frac{4 m a}{\eta^{2}},
$$

and

$$
\beta=-\frac{4 m b}{\eta^{2}}
$$

approximately. For the reasons given at the end of section 2 the component of angular momentum in the direction of the incoming particle does not appear in the scattering angles. 


\section{Discussion}

The main purpose of this paper is to present the deflection formulas (3.6) and (3.7) for the scattering of a high speed particle in the Kerr gravitational field. When $b=0$ the formula (3.6) agrees with the small angle of deflection of a photon moving in the equatorial plane of the Kerr source calculated by Boyer and Lindquist [13. This formula has been calculated in [1] and it has been commented on in some detail there. An important by-product of the

present work is the derivation of the light-like boost of the Kerr gravitational field in an arbitrary direction given in section 2 .

\section{Acknowledgment}

One of us (P.A.H.) thanks the C.N.R.S. for a position of Chercheur Associé.

\section{References}

[1] C. Barrabès and P. A. Hogan, Class. and Quantum Gravity 21, 405 (2004).

[2] D. M. Eardley and S. B. Giddings, Phys. Rev. D66, 044011 (2002); H. Yoshino and Y Nambu, Phys. Rev. D66, 065004 (2002).

[3] C. Barrabès and P. A. Hogan, Singular Null Hypersurfaces in General Relativity: light-like signals from violent astrophysical events (World Scientific, Singapore, 2004), p.79.

[4] R. P. Kerr, Phys. Rev. Lett. 11, 237 (1963).

[5] R. P. Kerr and A. Schild, Atti del Convegno sulla Relatività Generale: Problemi dell Energia e Onde Gravitazionali (Anniversary Volume, Fourth Centenary of Galileo's Birth), edited by G. Barbéra (Comitato Nazionale per le Manifestazione Celebrative, Firenze, 1965), p. 173; Applications of Nonlinear Partial Defferential Equations in Mathematical Physics, Proceedings of Symposia in Applied Mathematics (American Mathematical Society, Providence, RI, 1965), vol. XVII, p. 199.

[6] C. Barrabès and P. A. Hogan, Phys. Rev. D67, 084028 (2003).

[7] C. Barrabès and P. A. Hogan, Phys. Rev. D64, 044022 (2001). 
[8] Ya. B. Zel'dovich and I. D. Novikov, Relativistic Astrophysics Volume 1, Stars and Relativity (The University of Chicago Press, Chicago, 1978) p. 138.

[9] S. Weinberg, Gravitation and Cosmology (John Wiley, New York, 1972), p. 244.

[10] J. L. Synge and A. Schild, Tensor Calculus (University of Toronto Press, Toronto, 1964), p. 244.

[11] P. C. Aichelburg and R. U. Sexl, Gen. Relativ. Gravit. 12, 303 (1971).

[12] H. Balasin and H. Nachbagauer, Class. and Quantum Gravity 13, 731 (1996).

[13] R. H. Boyer and R. W. Lindquist, J. Math. Phys. 8, 265 (1967). 